\title{
EXPERIMENTAL AND NUMERICAL STUDY OF PULSATING TRANSVERSAL JETS
}

\author{
M. A. Goldfeld ${ }^{1}$, N. N. Fedorova ${ }^{1,2}$, I. A. Fedorchenko ${ }^{2}$, \\ G. A. Pozdnyakov ${ }^{1}$, K. Yu. Timofeev ${ }^{1}$, \\ and Yu. V. Zhakharova ${ }^{1,2}$ \\ ${ }^{1}$ Khristianovich Institute of Theoretical and Applied Mechanics \\ Siberian Branch of the Russian Academy of Sciences \\ 4/1 Institutskaya Str., Novosibirsk 630000, Russia \\ ${ }^{2}$ Novosibirsk State University of Architecture and Civil Engineering (Sibstrin) \\ 113 Leningradsksaya Str., Novosibirsk, Russia
}

\begin{abstract}
Paper presents results of joint experimental and numerical investigation of pulsating jet penetration into still air and supersonic flow. Goal of the study is to investigate two-dimensional (2D) Hartmann generator (HG) properties and clear up its possibilities in providing better mixing between air and secondary (injected) gases.
\end{abstract}

\section{INTRODUCTION}

Rising demands of modern aerodynamics to aircraft design efficiency place great emphasis on flow control technology. On this evidence, it is important to provide active control over large-scale turbulent structures which are known to be dominating in affecting separation, mixing, and noise generation in free and channel flows [1]. Recently, significant focus has been made on investigation of gas jets including synthetic ones, considering their utility as fluid actuator to control flows under different conditions.

Previous investigations have shown promising potential of the jet actuators in the high-speed flow control. It has been established that width of shear layers at high Reynolds number jets can be increased by an unsteady impact with amplitudes much higher than those used conventionally [2]. High Reynolds shear layers can also be intensified by oscillations of frequencies essentially greater than the frequencies of their own instability and of larger amplitudes [3]. This type of investigations has been performed for a cavity flow with a strong resonance. It is not known till now whether this situation can be realized for a shear layer of broadband spectrum.

This is an Open Access article distributed under the terms of the Creative Commons Attribution License 4.0, which permits unrestricted use, distribution, and reproduction in any medium, provided the original work is properly cited. 
There are researches on an HG application to the flow structure control [25]. The HG consists of a nozzle and a channel fixed opposite to each other. The nozzle jet interacts with the channel entrance in an unstable mode and it leads to flow oscillations at some frequency. At first, the features of the amplitude frequency response of the $\mathrm{HG}$ and the way it is influenced by different parameters including the channel depth, the distance between the nozzle and the channel entrance, and the jet Mach number (nozzle pressure ratio) have been studied by Hartmann and Trolle [6]. Sprengler [7] noticed that in the closed part of the channel, an intense gas heating can take place under the action of a sequence of weak shocks being trapped in the channel. He has also mentioned that subsonic jets could heat gas in the channel as well. Hereafter, this study of the Hartmann-Sprengler (HS) generator became a subject of numerous experimental and theoretical investigations [8-16]. Most of the works have been done under nonuniform flow conditions downstream the convergent sonic nozzle exit [8-10]. At the same time, the resonance has been obtained for perfectly expanded supersonic jets $[4,5]$. Moreover, experiments with the HS generator have been carried out in a confined space to study oscillations and flow structures in the tubes placed in the channel at subsonic [12] and supersonic [13] flow speeds.

In practice, such actuators have been already utilized for separated flow control [14], cavity flow control $[3,4,17]$, and jet collision [5]. Possibilities of HG implementation for fuel and air mixing at supersonic combustion chambers have been considered as well $[18,19]$.

This paper differs from the most of the previous works in several aspects. First, the channel producing the jet is a narrow slot and not a circular orifice as in the large majority of the investigations. Second, flow nonuniformities propagate through the slot channel in one direction instead of an axisymmetry model. Therefore, the shear layer has different parameters at the internal and external surfaces.

It has been demonstrated [9] that convergent stream impingent on an open tube exit can result in three different oscillating modes: jet instability mode, regurgitant mode, and screech mode. The jet instability mode is observed for subsonic jets and is accompanied by a periodic vortex structure generation in the jet mixing layer. The instability frequency is defined by the Strouhal number of the vortex. Both the regurgitated and the screech modes appear in nonuniform supersonic flow generated by the underexpanded jet and the mode type depends on the distance between the nozzle exit and the open channel entrance, the exit velocity, and the jet pressure ratio. The screech mode takes place when the channel is in unstable mode under adverse axial pressure gradient as observed by Hartmann and Trolle [6]. The supersonic flow deceleration leads to the normal shock generation in front of the channel entrance and then the oscillation frequency is determined by local flow conditions. It is obvious that the mode type, instability parameters, and their frequency response can be greatly different in axially symmetric and plane flows. 
The main goal of the current work is to investigate the 2D HG and to determine its frequency response both numerically and experimentally. A numerical validation is provided and a study of gasdynamic parameters of the flow on the flow modes is performed.

\section{EXPERIMENTAL SETUP AND APPARATUS}

Experimental model represents a flat plate of 100-millimeter width with a sharp leading edge. In the middle part of it, a slot generator is installed. The model sketch and its principal sizes are shown in Fig. 1. All blocks of the generator are exchangeable to provide a possibility for modifying the geometry of the inner dummy plane channel and the distance between the channel entrance and the nozzle exit. Special inserts allow changing geometrical parameters of a stagnation chamber ahead of the nozzle and nozzle dimensions to achieve sonic or supersonic speed at the exit. The model is installed on a pylon to fit a test section of the aerodynamic facility. It permits to investigate free flow influence on the disturbance propagation and the flow structure over the model surface under the action of the HG.

A supply pipeline connects the stagnation chamber with a compressed gas source of total pressure value up to $10 \mathrm{MPa}$. Both air and helium are used in the experiments. Later, hydrogen and argon are planned to be used. During the runs, the total pressure measurements at the nozzle entrance are made, along with the total pressure records by means of Pitot probes at the distance of 15, 30, and $45 \mathrm{~mm}$ downstream of the channel entrance and static pressure registration

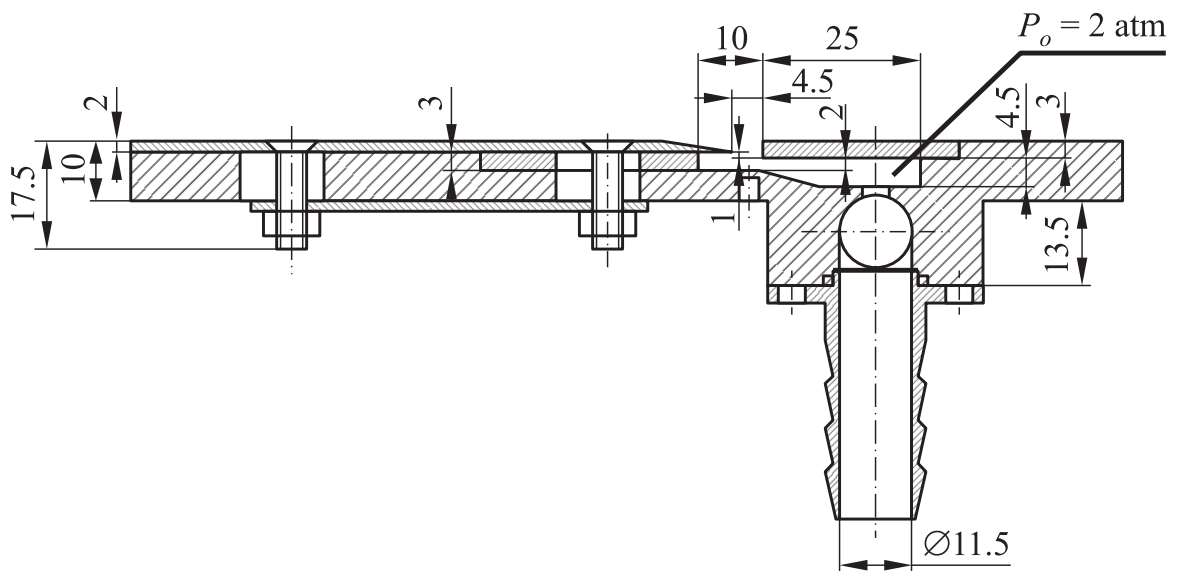

Figure 1 Model sketch. Dimensions are in millimeters 
in a cavity between the nozzle and the channel (resonator). The amplitude frequency response has been measured with piezoelectric pressure probes installed in the cavity and on the top resonator wall at the distance of $10 \mathrm{~mm}$ from the entrance. In the tests with the external flow, the total and static pressures are registered at the nozzle exit and the static pressure before of the HG.

\section{MATHEMATICAL MODEL AND NUMERICAL APPROACH}

Unsteady full averaged Navier-Stokes equations in 2D formulation are used in this work for modeling the problem of jet injection. The Navier-Stokes equations are closed with the $k-\omega$ shear stress transport (SST) turbulence model. In the case of a mixture flow (air and injected gas), the equation of conservation of the injected gas mass fraction with allowance for turbulent diffusion is used. The calculations are performed with the aid of Computational Fluid Dynamics (CFD)

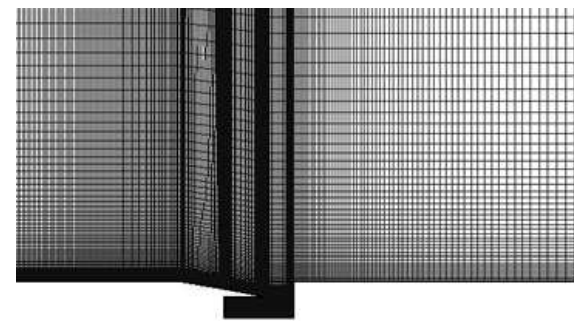

Figure 2 Computation grid
ANSYS Fluent 14.0 software. Spatial discretization of convective terms is performed by the high-order Roe schemes [20]. Implicit formulation of first-order accuracy is implemented for the nonsteady integration in time. A structured computational grid with refinement in vicinity of the $\mathrm{HG}$ and the plate surface is used (Fig. 2). At least, eight nodes are put on the inlet width.

\section{Validation of the Mathematical Approach}

The mathematical model and algorithm are tested against the experimental data [21] on slotted injection of nitrogen jets from the plate surface into a supersonic air flow with Mach number $\mathrm{M}_{\infty}=2.61$. At the center of the plate whose width is $457 \mathrm{~mm}$, there is a slot $0.27 \mathrm{~mm}$ wide. The computations are performed for several experimentally studied cases under the conditions described in Table 1. A computation has been performed on a sequence of refined grids. Grid refinement has been performed by using a function of adaption in terms of the pressure gradient of the ANSYS Fluent software. The pressure distributions on the wall obtained on four meshes with different numbers of nodes $\left(77 \cdot 10^{3}\right.$, $104 \cdot 10^{3}, 133 \cdot 10^{3}$, and $174 \cdot 10^{3}$ ) show that the difference in results decreases from $3 \%$ to $1 \%$ as the number of nodes is increased.

Figure $3 a$ shows the calculated Mach number fields. The nitrogen jet escaping from the slot forms a supersonic flow region 1, which is typical for underexpanded 
Table 1 Parameters of the jet and external flow [21]

\begin{tabular}{ccccc}
\hline Flow domain & $C_{\mathrm{N}_{2}}$ & $P, \mathrm{kPa}$ & $\mathrm{M}$ & $T_{0}, \mathrm{~K}$ \\
\hline External flow & 0 & 6.8 & 2.61 & 988 \\
Jet & 1 & $84.5,158.7,302.7$ & 1 & $943,935,928$ \\
\hline
\end{tabular}

supersonic jets $[22,23]$. The velocity of the flow bounded by the contact surfaces of the jet increases, and compression waves reflected from these surfaces generate internal shocks. After that, a strong transverse shock 2 (Mach disk) is formed, and the flow velocity behind this shock becomes subsonic. The jet serves as an obstacle for the supersonic flow, which results in a shock wave 5 and turbulent boundary layer 3 separations. Above the recirculation zone 4, one can see

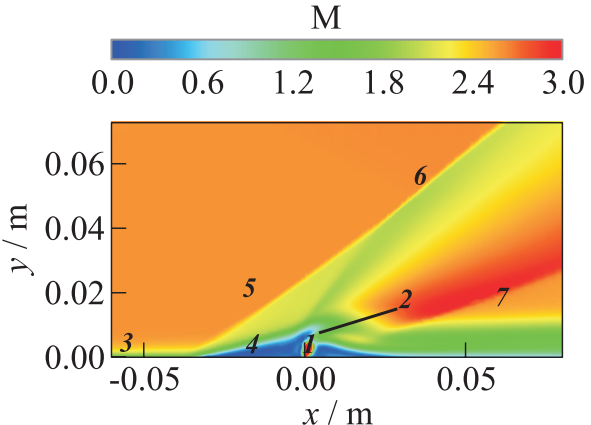

(a)

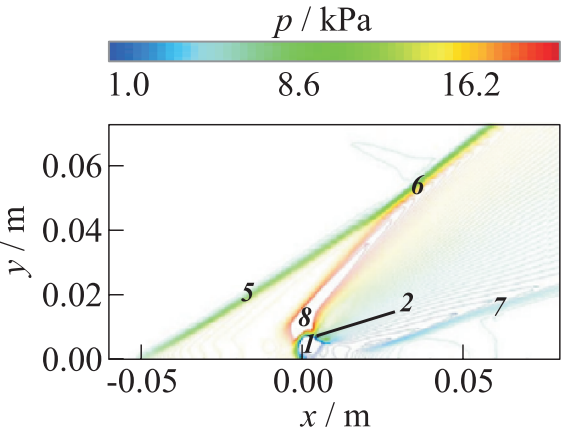

(b)

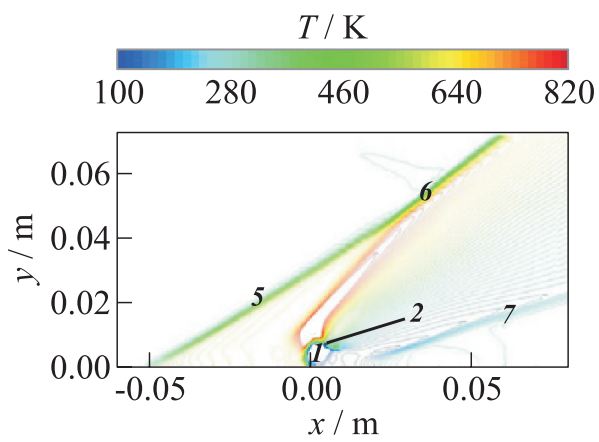

(c)

Figure 3 Mach number $(a)$, pressure $(b)$, and temperature $(c)$ contours in the case of nitrogen injection jet into an air flow at $\mathrm{M}_{\infty}=2.61$ : 1 - supersonic flow; $2-$ Mach disk; 3 - separation of the turbulent boundary layer; 4 - recirculation zone; 5 - separation shock; 6 - jet-induced shock wave; 7 - reattachment shock; and 8 local pressure peak 


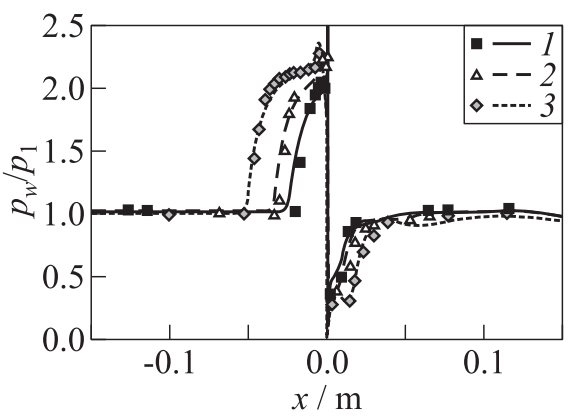

Figure 4 Calculated (curves) and experimental [21] (signs) distributions of pressure on the plate $p_{w}$ for different ratios of pressures in the external flow and injected nitrogen: $1-p_{0} / p_{1}=23.5 ; 2-p_{0} / p_{1}=44.5$; and $3-p_{0} / p_{1}=82.9$

the separation shock 5 and the jetinduced shock wave 6 . The separation region behind the jet is tailed by the reattachment shock 7 .

Figure $3 b$ shows the static pressure contours. The maximum deceleration of the flow is observed in the vicinity of the frontal edge of the jet, where a local pressure peak 8 is formed owing to meeting of two oppositely directed flows. The maximum temperature $T=950 \mathrm{~K}$ is reached near the frontal separation point (Fig. 3c).

Figure 4 shows the experimental and calculated pressure distributions on the plate surface at $\mathrm{M}_{\infty}=2.61$ for nitrogen jets. It is seen that the pressure values and the separation region lengths are in good agreement both ahead of the jet and behind it. As the jet injection pressure increases, the jet-flow interaction intensifies and the separation region length increases.

\section{PROBLEM STATEMENT AND BOUNDARY CONDITIONS}

Both air and helium jets injected into a cavity opened to an external semibounded space are considered as used in the experiments. Two cases are considered:

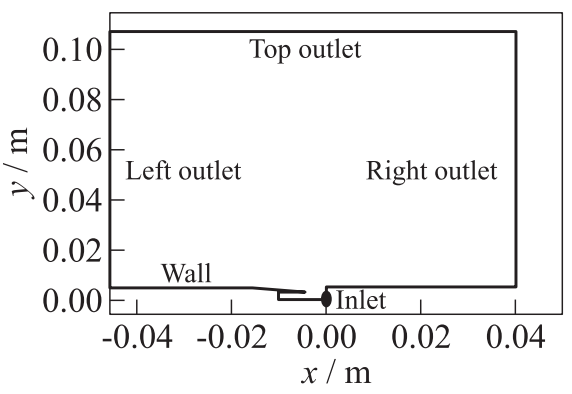

(a)

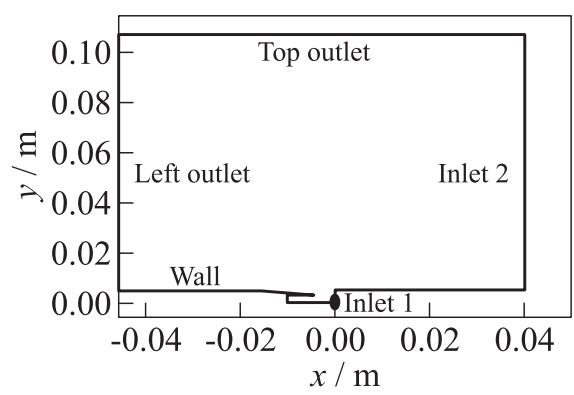

(b)

Figure 5 Computational domain for case of the Hartmann generator without external flow $(a)$ and with supersonic external flow $(b)$ 
a jet immersed into still air and injected into a supersonic external flow. The corresponding $2 \mathrm{D}$ computational domain and boundary conditions for both cases are shown in Figs. $5 a$ and $5 b$. In the first case (see Fig. $5 a$ ), at the inlet boundary, sonic conditions for air or helium injection are specified at total temperature $T_{0}=300 \mathrm{~K}$ and different total pressures of 2 and $4 \mathrm{~atm}$. In the second case, the inlet 2 boundary condition consists of air flowing from left to right at Mach number 1.1 (see Fig. $5 b$ ).

Static pressure, Mach number, and static temperature values are specified at the inlet boundaries. Adiabatic no-slip boundary conditions are used at the walls, and specified pressure and total temperature values are applied at the outlet sections of the domain.

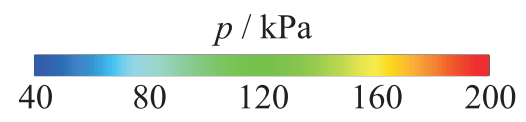

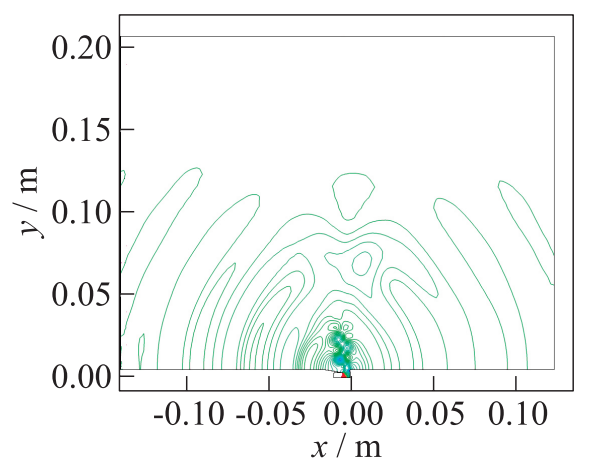

(a)

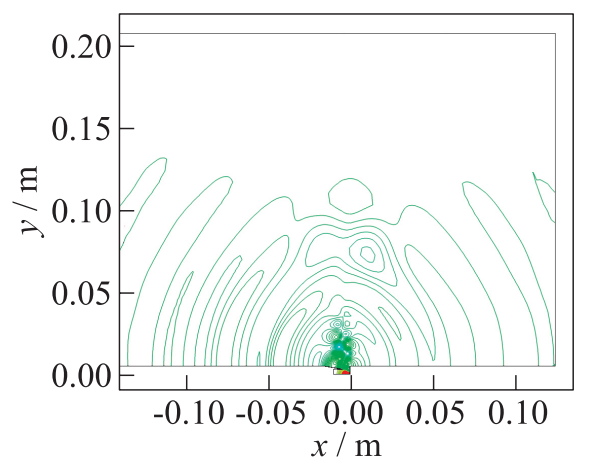

(c)

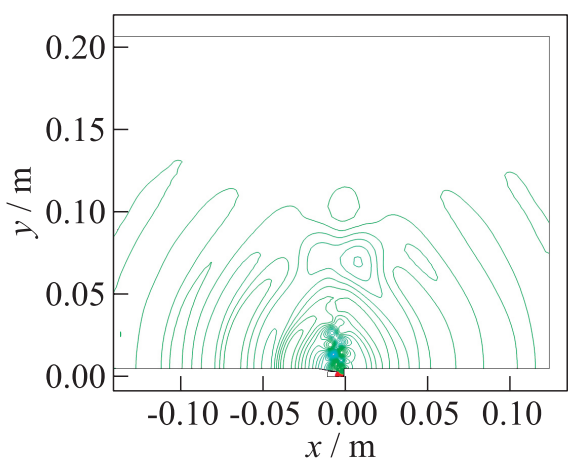

(b)

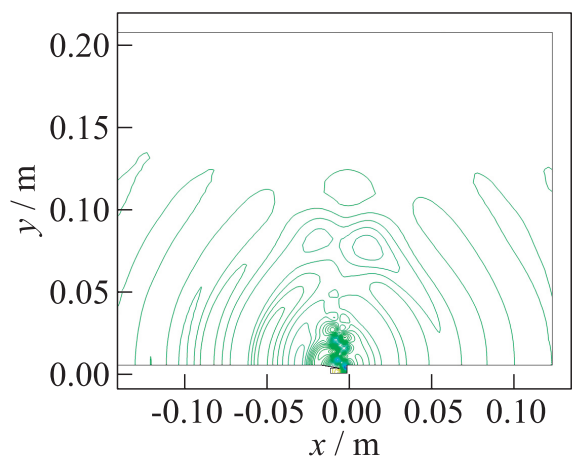

(d)

Figure 6 Pressure contours for the immersed air jet at different time moments 


\section{RESULTS AND DISCUSSION}

\subsection{Jet Injection into Still Space}

First, a sonic air jet injection into still air is investigated. In this case, nonsteady behavior of the jet inside the Hartmann tube cavity leads to the acoustic field generation, and fast Fourier transformation of a signal of the total mass flow calculated over the outlet boundaries allows for determining the frequency response of the process.

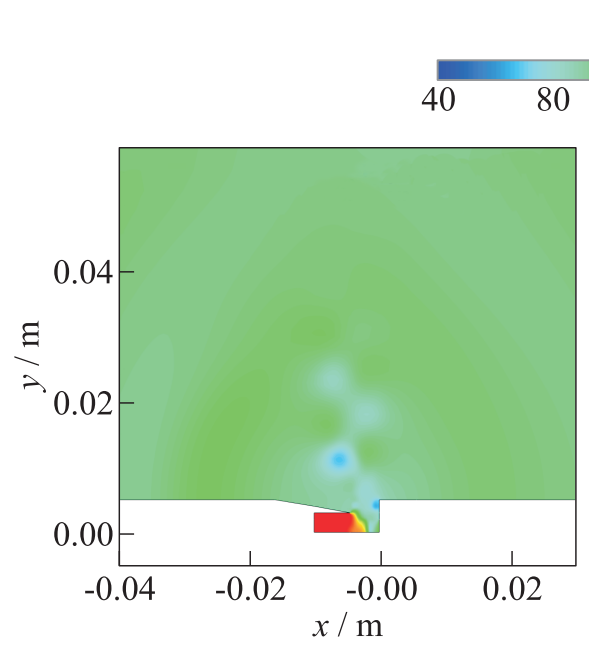

(a)

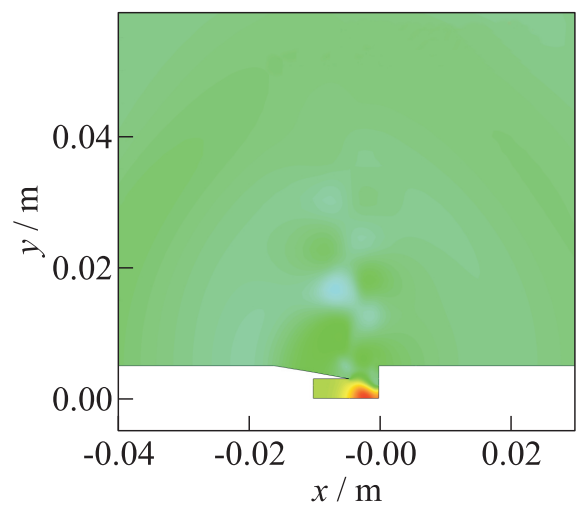

(c) $p / \mathrm{kPa}$

$120 \quad 160 \quad 200$

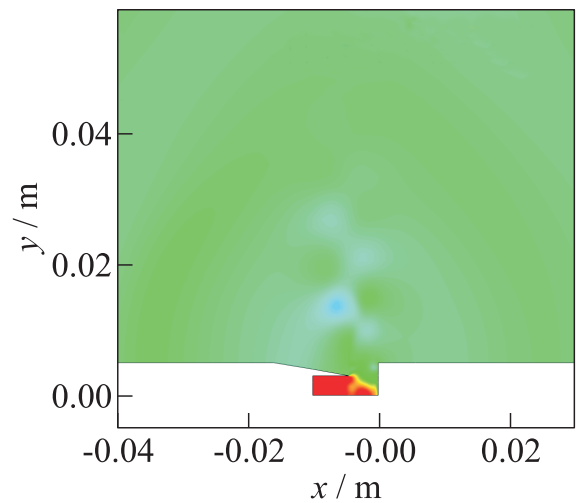

(b)

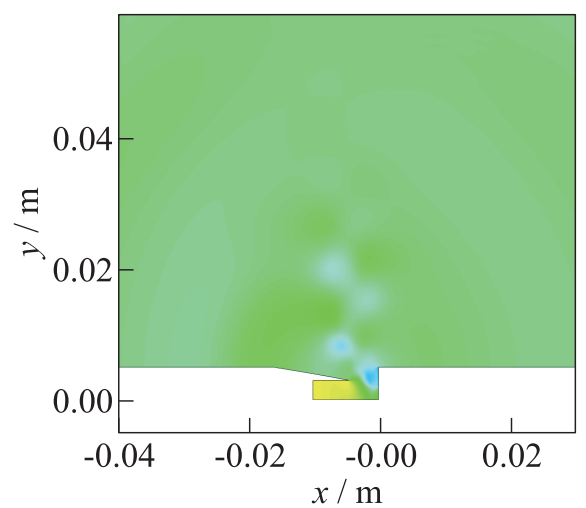

(d)

Figure 7 Pressure fields for the immersed air jet at different time moments: zoomed pictures 
Several pressure contours at different time moments with period of $25 \mathrm{~s}$ are presented in Fig. 6. It can be observed that the pressure wave generation takes place and the waves propagate from the source (HG) toward the outlets. Zoomed pressure fields in vicinity of the generator (Fig. 7) show behavior of the shock generated because of jet injection from a nozzle into the cavity.

A high-pressure zone is formed inside the dummy part of the channel (Figs. $7 a$ and $7 b$, red domains) and it results in the jet displacement to the upper space. Then, the pressure level inside the cavity decreases and a new portion of the injected gas is sucked in the cavity (Figs. $7 c$ and $7 d$ ). After that, the process repeats organizing oscillations of the external flow.

Power spectral densities (PSD) of the process signal obtained both numerically and experimentally are shown in Figs. $8 a$ and $8 b$, respectively. It can be seen that the main flow frequency $11 \mathrm{kHz}$ is achieved both in the simulated and experimental data. The frequency responses at two different total pressures of the jet of 2 and 4 bar have been modeled as shown in Fig. $8 a$. It can be seen that the higher total pressure at the inlet leads to the higher frequency of the oscillations.

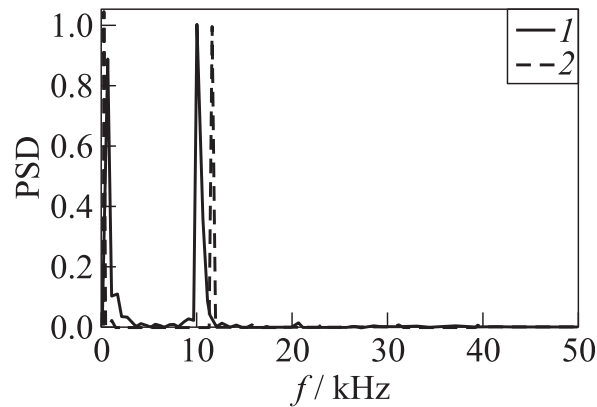

(a)

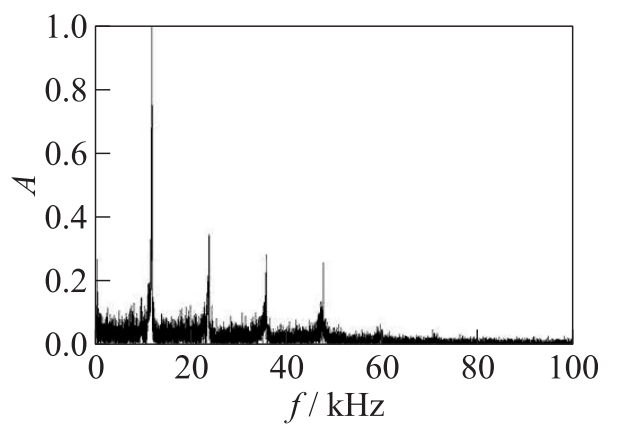

(b)

Figure 8 Power spectral density distributions for the air sonic immersed jet obtained numerically $(a)\left(1-p_{0}=2\right.$ at; and $2-p_{0}=4$ at $)$ and experimentally $(b)$

\subsection{Helium Jet Injection into Still Air}

Helium jet injection into still air under the same gasdynamic conditions has been investigated as well. Dynamics of the helium jet evolution is shown in Fig. 9. At the last two moments (Figs. $9 c$ and $9 d$ ), oscillation influence on the jet structure becomes visible, vortex shedding occurs, and the jet flow mixes with the air.

The PSD distributions for the pressure records at the plate surface and inside the cavity along with the mass flow registration are obtained numerically and shown in Fig. 10a. While the plate pressure signal and the mass flow record give 
$\mathrm{He}$

$\begin{array}{llll}0 & 0.33 & 0.66 & 0.99\end{array}$

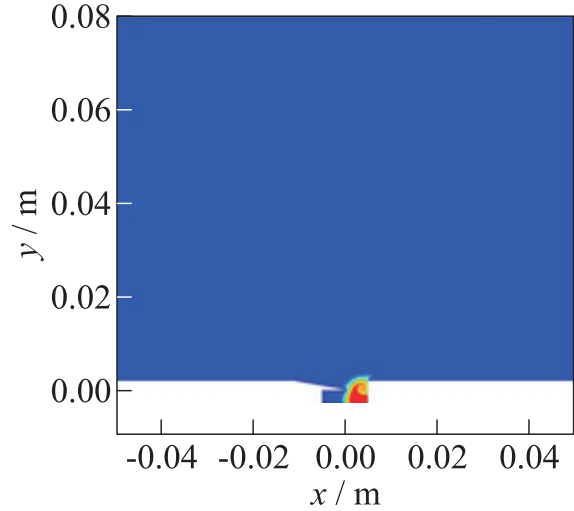

(a)

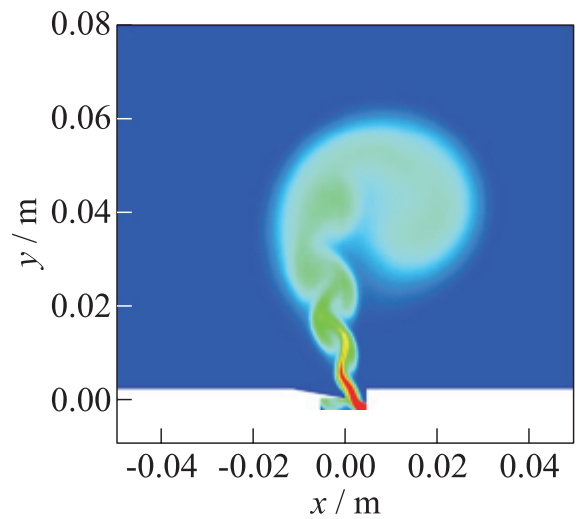

(c)

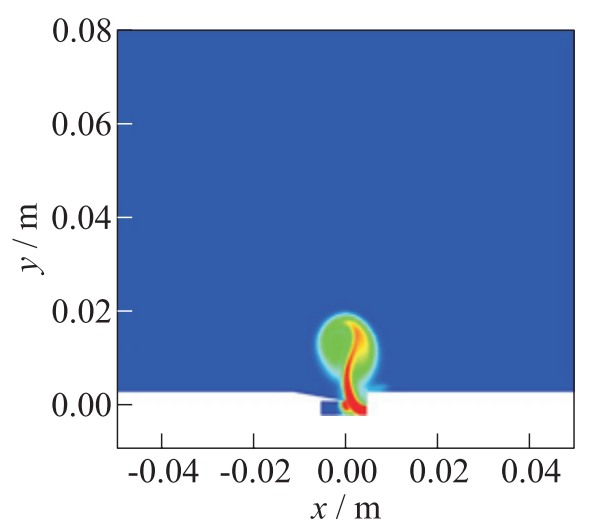

(b)

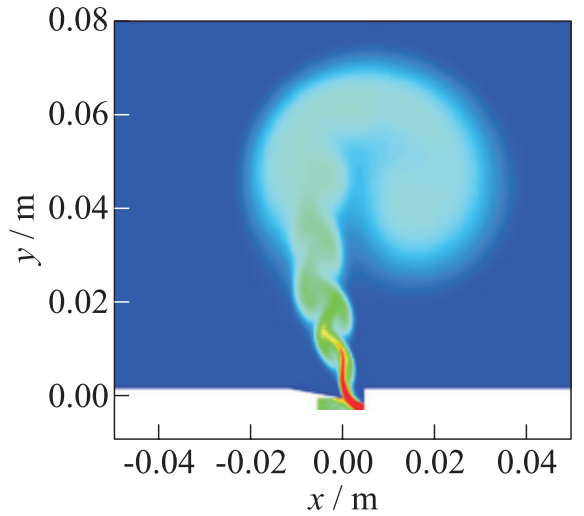

(d)

Figure 9 Helium mass fraction fields for the Helium sonic immersed jet obtained at different time moments

the same frequency value, the pressure record inside the cavity produces a higher frequency response of $27.8 \mathrm{kHz}$ which agrees better with the first experimental value of $32 \mathrm{kHz}$ (Fig. 10b).

\subsection{Air Jet Injection into Supersonic External Flow}

At the third stage, an air jet injection into a supersonic external flow is investigated. Mach number and total pressure of external flow equal 1.1 and $2 \mathrm{~atm}$, 


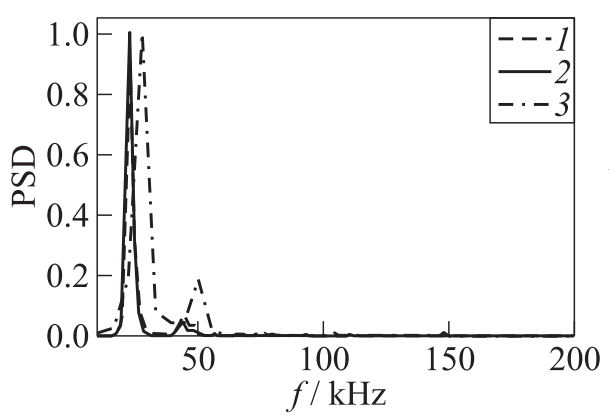

(a)

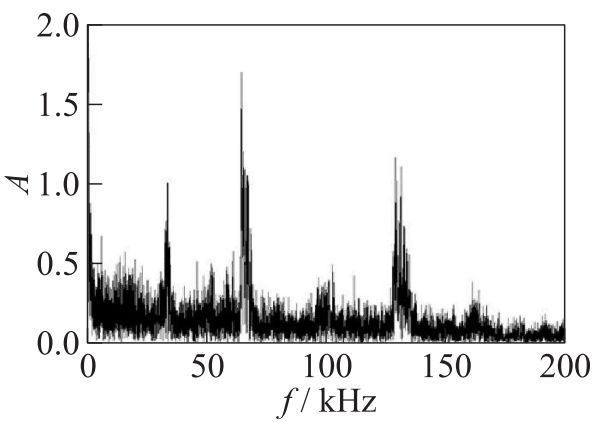

(b)

Figure 10 Power spectral density distributions for the helium sonic immersed jet obtained numerically (a) (1 - massflow; 2 - plate; and 3 - cavity) and experimentally $(b)$

respectively. Frequency response formation is the same as it was described above for the case of jet injection into still space.

Power spectral density of the process signal is shown in Fig. 11. The main flow frequency of about $10 \mathrm{kHz}$ is achieved in the simulated data (Fig. 11a) which is similar to the case of jet injection into still air. The results of the experimental data show two main flow frequencies 10 and $16 \mathrm{kHz}$ (Fig. 11b).

Pressure fields for the different time moments are shown in Fig. 12. A significant change of the flow structure and pressure level inside the Hartmann cavity tube was obtained.

At the initial time moment, low-pressure level can be observed (Fig. 12a) inside the Hartmann cavity tube.

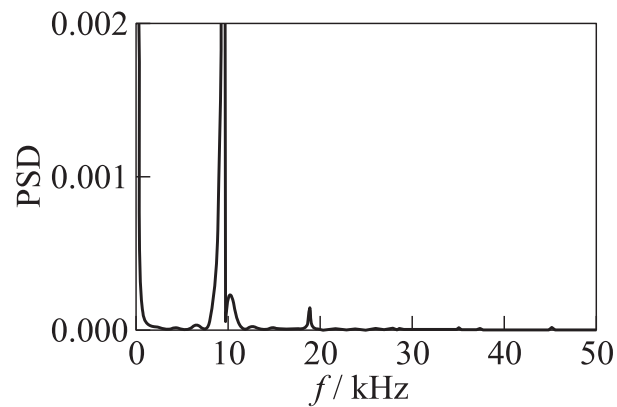

(a)

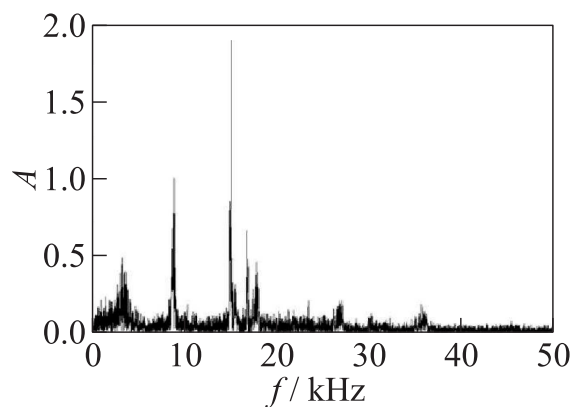

(b)

Figure 11 Power spectral density distributions for the air sonic jet injected into supersonic external flow obtained numerically $(a)$ and experimentally $(b)$ 


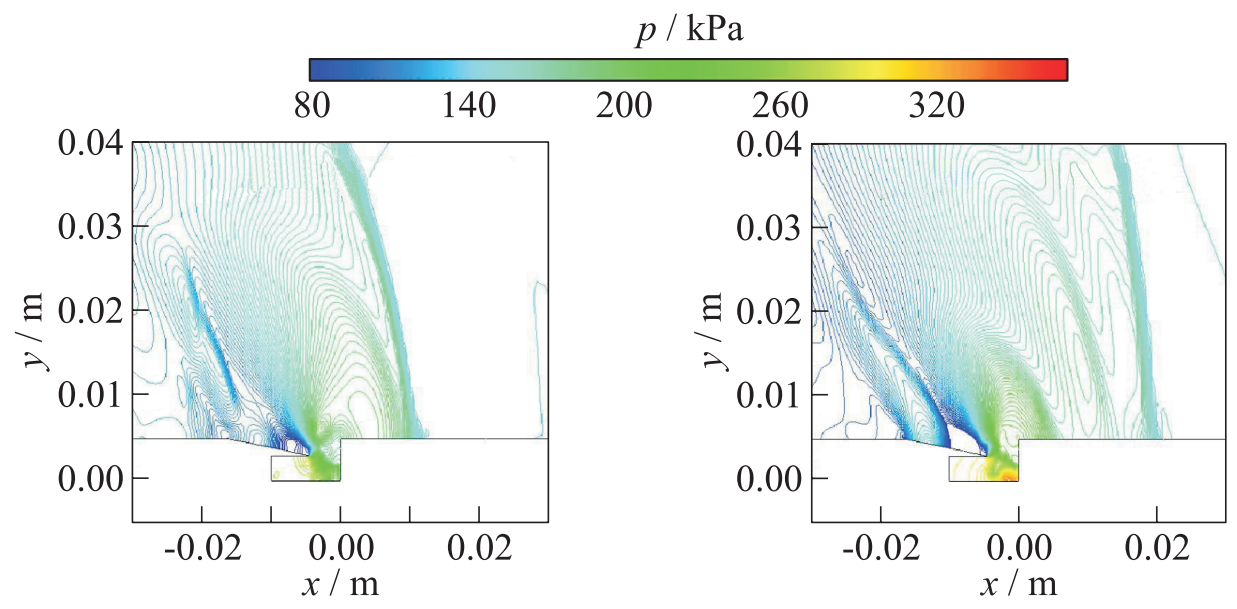

(a)

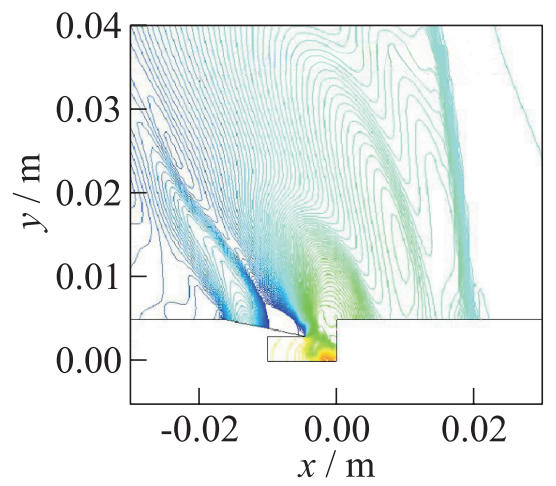

(c)

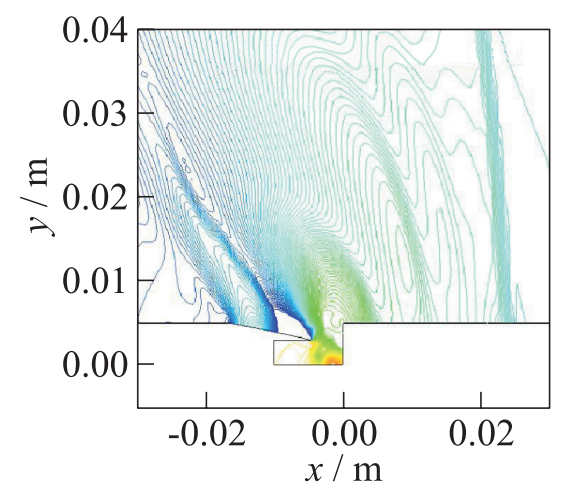

(d)

Figure 12 Pressure fields for the jet injection in supersonic external flow

After more injected gas is introduced into the cavity, the pressure level increases (Fig. 12b). The complex wave structure is formed due to the interaction of the jet with an external flow. The boundary layer formed on the plate before inlet 1 is separated.

The bow shock is formed before the jet at the initial time moment (Figs. $12 c$ and $12 d)$. With time, the shock moves upstream till some position close to the inlet 2 boundary.

As a result, the external flow becomes subsonic after the bow shock (Fig. 13). 


\section{CONCLUDING REMARKS}

Joint computational and experimental research of pulsating transversal air and helium jets in still air and supersonic flow are carried out. Comparison of experimental and simulated results on frequency response has shown a good agreement.

The results of jet injection into still space for two values of pressure have shown that an increase in pressure leads to an increase of frequency response.

The presence of an external flow does not lead to a significant change in the frequency response by comparison with cases for still air.

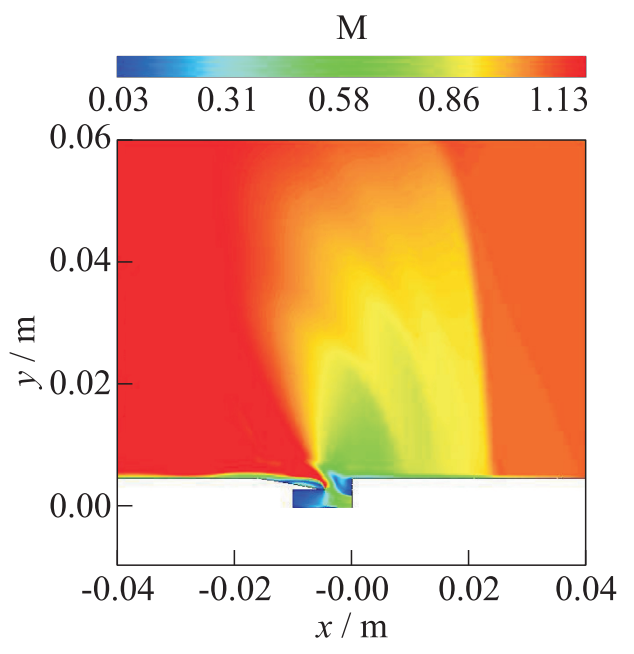

Figure 13 Mach number field for the jet injection in supersonic external flow

\section{ACKNOWLEDGMENTS}

This work was supported by the Russian Foundation for Basic Research (Grant Nos. 12-07-00571-a and 12-08-00955-a) and by the Ministry of Science and Education (Contract No.14.B37.21.1553).

\section{REFERENCES}

1. Papamoschou, D., and A. Roshko. 1988. The turbulent compressible shear layer: An experimental study. J. Fluid Mech. 197:715-816.

2. Kibens, V., J. Dorris III, D. M. Smith, and M. F. Mossman. 1999. Active flow control technology transition: The Boeing ACE Program. AIAA Paper No. 99-3507.

3. Stanek, M. J., G. Raman, V. Kibens, and J. Ross. 2000. Control of cavity resonance through very high frequency forcing. AIAA Paper No. 2000-1905.

4. Raman, G., V. Kibens, A. Cain, and J. Lepicovsky. 2000. Advanced actuator concepts for active aeroacoustic aontrol. AIAA Paper No. 2000-1930.

5. Raman, G., and V. Kibens. 2001. Active flow control using integrated powered resonance tube actuators. AIAA Paper No. 2001-3024.

6. Hartmann, J., and B. Trolle. 1927. A new acoustic generator. J. Sci. Instrum. $4(4): 101-111$. 
7. Sprenger, H. 1954. Ueberthermische Effekte in Resonanzrohen. Mitteilungen aus dem Institut fur Aerodynamik. Zurich: E.T.H. 21:18-35.

8. Brocher, E., C. Maresca, and M.-H. Bournay. 1970. Fluid dynamics of resonance tube. J. Fluid Mech. 43(2):369-384.

9. Sarohia, V., and L. H. Back. 1979. Experimental investigation of flow and heating in a resonance tube. J. Fluid Mech. 94(4):649-672.

10. Iwamoto, J. 1986. Necessary conditions for starting and maintaining a stable oscillatory flow in a Hartmann-Sprenger tube. In: Flow visualization IV. Washington, D.C.: Hemisphere. 507-512.

11. Brocher, E., and E. Duport. 1988. Resonance tubes in a subsonic flowfield. AIAA J. 26(5):548-552.

12. Ko, S.-M., and K.-S. Chang. 1998. Resonant pulsatile flows of a HartmannSprenger tube. Int. J. Comput. Fluid D 6(4):439-452.

13. Kastner, J., and M. Samimy. 2002. Development and characterization of Hartmann tube fluidic actuators for high-speed flow control. AIAA J. 40(10):1926-1934.

14. Hamed, A., K. Das, and D. Basu. 2003. Numerical simulation and parametric study of Hartmann-Sprenger tube based power devices. AIAA Paper No. 2003-0550.

15. Raman, G., A. Mills, and V. Kibens. 2004. Development of powered resonance-Tube acutators for aircraft flow control applications. J. Aircraft 41(6):1306-1314.

16. Xia, G, D. Li, and C. L. Merkle. 2007. Effects of a needle on shrouded HartmannSprenger tube flows. AIAA J. 45(5):1028-1035.

17. Kim, K.-H., and K.-S. Chang. 1994. Three-dimensional structure of a supersonic jet impinging on an inclined plate. J. Spacecraft Rockets 31(5):778-782.

18. Bogdanoff, D.W. 1994. Advanced injection and mixing techniques for scramjet combustors. J. Propul. Power 10(2):183-190.

19. Murugappan, S., and E. Gutmark. 2004. Flowfield and mixing control of an underexpanded jet. AIAA J. 42(8):1612-1621.

20. Roe, R. L. 1986. Characteristic based schemes for the Euler equations. Annu. Rev. Fluid Mech. 18:337-365.

21. Zukoski, E. E., and F. W. Spaid. 1964. Secondary injection of gases into a supersonic flow. AIAA J. 2(10):1689-1696.

22. Schetz, J.A., P.F. Hawkins, and H. Lehman. 1967. Structure of highly underexpanded transverse jets in a supersonic stream. AIAA J. 5:882-884.

23. Dulov, V.G., and G. A. Luk'yanov. 1984. Gas dynamics of exhaustion processes. Novosibirsk: Nauka. 236 p. [In Russian.] 